\title{
Experimental validation of fluid inertia models for a cylinder settling in a quiescent flow
}

\author{
F. Cabrera $\odot,{ }^{1}$ M. Z. Sheikh, ${ }^{1,2}$ B. Mehlig, ${ }^{3}$ N. Plihon, ${ }^{1}$ M. Bourgoin, ${ }^{1}$ A. Pumir, ${ }^{1,4}$ and A. Naso $\oplus^{5}$ \\ ${ }^{1}$ Univ Lyon, ENS de Lyon, Univ Claude Bernard Lyon 1, CNRS, \\ Laboratoire de Physique, F-69342 Lyon, France \\ ${ }^{2}$ Department of Mechanical Engineering, University of Engineering and Technology, 54890, Lahore, Pakistan \\ ${ }^{3}$ Department of Physics, Gothenburg University, 41296 Gothenburg, Sweden \\ ${ }^{4}$ Max-Planck Institute for Dynamics and Self-Organization, Göttingen, D-37077, Germany \\ ${ }^{5}$ Univ Lyon, CNRS, Ecole Centrale de Lyon, INSA Lyon, Univ Claude Bernard Lyon 1, \\ Laboratoire de Mécanique des Fluides et d'Acoustique, UMR 5509, 69130 Ecully, France
}

(Received 15 July 2021; accepted 24 January 2022; published 3 February 2022)

\begin{abstract}
The precise description of the motion of anisotropic particles in a flow rests on the understanding of the force and torque acting on them. Here, we study experimentally small, very elongated particles settling in a fluid at small Reynolds number. In our experiments, we can, to a very good approximation, relate the rate of rotation of cylindrical tungsten rods, of aspect ratios $\beta=8$ and $\beta=16$, settling in pure glycerol, to the torque they are experiencing. This allows us to compare the measured torque with expressions obtained either in the slender-rod limit or in the case of spheroids. Both theories predict a simple angle dependence for the torque, which is found to capture very well the experimental results. The slender-rod theory overestimates the results for the two aspect ratios considered, while the expression obtained for a spheroid provides a better approximation for $\beta=16$. Comparing our results with those of previous experiments provides further insight on the conditions of validity of the slender-rod theory. The translational dynamics is shown to be in qualitative agreement with the slender-rod and spheroid models, the former one being found to represent better the experimental data.
\end{abstract}

DOI: 10.1103/PhysRevFluids.7.024301

\section{INTRODUCTION}

The settling of nonspherical particles at low Reynolds number in a quiescent fluid is a subtle problem. It has been known for a long time that describing the angular degrees of freedom with the Stokes approximation leads to an indeterminacy in the settling angle: the particle may settle at any angle with respect to the vertical. This degeneracy, however, is lifted by the action of the fluid, already in the limit of a small but nonzero particle Reynolds number $\operatorname{Re}_{p}$. Whereas the correction to the translational velocity has been understood for a long time, the torque acting on a nonspherical particle is not as well understood. Cox [1] determined the torque acting on nearly spherical objects. Later, Khayat and Cox [2] determined the torque for slender particles. The exact conditions of validity of this asymptotic prediction are, however, a bit unclear. More recently, the torque acting on spheroids was determined for spheroids of arbitrary aspect ratio [3].

Because of the importance of the problem, many contributions have been devoted to its numerical solution, to determine approximate torque parametrizations as a function of the particle Reynolds number $\operatorname{Re}_{p}$ and particle shape [4-10]. The effect of small to moderate Reynolds numbers for spheroids over a large range of aspect ratios $\beta$ was recently analysed by means of numerical simulations [11], allowing a direct comparison with the small- $\operatorname{Re}_{p}$ theory [3] in the limit of small 
$\mathrm{Re}_{p}$. It was found that the theory [3] generally provides the correct functional form and the correct magnitude for the torque if $\operatorname{Re}_{p}$ is small enough. However, it tends to over predict the numerically determined values when $\operatorname{Re}_{p} \gtrsim 1$. The hydrodynamic loads on a fixed finite-length circular cylinder in a uniform flow have also been obtained numerically, from creeping-flow conditions to strongly inertial regimes [12]. Semi-empirical models based on theoretical predictions and incorporating finite-length and inertial corrections extracted from the numerical data were then derived. The authors suggest that the conditions $\operatorname{Re}_{D} \ll 1$ and $\beta \gg 1$ (where $\operatorname{Re}_{D}$ is the particle Reynolds number based on its diameter, i.e. $\operatorname{Re}_{D} \sim \operatorname{Re}_{p} / \beta$ ), on which the asymptotic prediction [2] is usually grounded, should rather be understood as: $\operatorname{Re}_{D} \ll 1$ and $\operatorname{Re}_{D} / \beta^{2}$ to be smaller than a small number estimated to be of the order of $10^{-3}$ [12]. In all these numerical investigations, the particle is kept fixed, with a given orientation, in a uniform flow. There have been, in comparison, far fewer experimental studies.

In experiments where rods settle through a simple vortical flow, it was shown that the effect of fluid inertia could not be ignored [13]. More recently, the force and torque measured in settling experiments of symmetric and asymmetric fibers roughly three times denser than the fluid were confronted to the model of Khayat and Cox [14]. For symmetric fibers, the expression of torque by Khayat and Cox overpredicts the torque experimentally measured for $\beta=20$ and $\operatorname{Re}_{p}=1.6$, while it predicts better the measurements for $\beta=100$ and $\operatorname{Re}_{p}=8.6$. Performing experiments in complementary regimes is particularly important to provide further constraints on the validity of existing theory. In particular, $\operatorname{Re}_{p}$ values smaller than those of Ref. [14] should enable to clarify the role of the particle Reynolds number on the applicability of the results of Khayat and Cox.

To this aim, we consider again the problem in a different setup, using small tungsten rods, roughly 12 times denser than the surrounding fluid, which is pure glycerol. The resulting particle Reynolds number, $|\mathbf{w}| l / v$, where $l$ is the half-length of the rod, $|\mathbf{w}|$ the norm of the center of mass velocity and $v$ the fluid viscosity, is always smaller than 0.39. Our experimental setup (based on 3D tracking of the particle rotation and translation) allows us to determine precisely all the degrees of freedom of the motion, and to deduce from it the torque acting on the particle. Our experimental results are then systematically compared to the predictions for slender-rods and spheroids $[2,3]$.

One motivation for studying the problem comes from the modeling of processes involving nonspherical particles settling in turbulent flows. This includes, in an engineering context, problems involving paper fibers [15]. The question is also particularly relevant in the environmental sciences, consider for example the settling of plankton in the oceans [16-18], or ice crystals settling in clouds [19-24].

The article is organized as follows. We first introduce in Sec. II the translational and rotational equations of motion for spheroids and slender-rods. The experimental design is then described in Sec. III. The results are presented in Sec. IV. Finally, our conclusions are summarized in Sec. V.

\section{THEORETICAL BACKGROUND}

Our experiments were performed with cylindrical rods of density $\rho_{p}$, half-length $l$ and radius $a$. The particle aspect ratio is $\beta=l / a$. The motion of the particles is characterized by the center of mass $\mathbf{x}$ and the unit vector $\hat{\mathbf{n}}$ characterizing the orientation, as illustrated in Fig. 1. The orientation of $\hat{\mathbf{n}}$ is defined by the angles $\psi$ and $\theta$. The vector $\hat{\mathbf{p}}$ is orthogonal to the projection of $\hat{\mathbf{n}}$ onto the horizontal $\left(\hat{\mathbf{e}}_{x}, \hat{\mathbf{e}}_{y}\right)$ plane, $\hat{\mathbf{p}}=\sin (\psi) \hat{\mathbf{e}}_{x}-\cos (\psi) \hat{\mathbf{e}}_{y}$, and $\hat{\mathbf{e}}_{z}=-\mathbf{g} /|\mathbf{g}|$, where $\mathbf{g}$ is gravity, is the vertical. In the experiments, the initial angular velocity was orthogonal to the plane spanned by $\mathbf{g}$ and $\hat{\mathbf{n}}$ (see Sec. IV). In other words, the angle $\psi$ remains constant, so the angular dynamics is described by $\theta$ only.

The particle Reynolds number is defined as

$$
\operatorname{Re}_{p}=\frac{l|\mathbf{w}|}{v},
$$


(a)

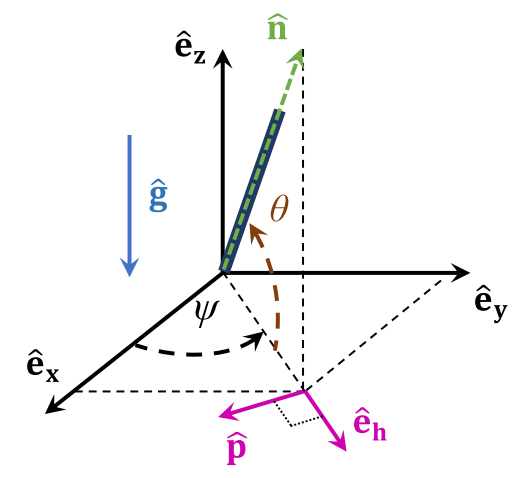

(b)

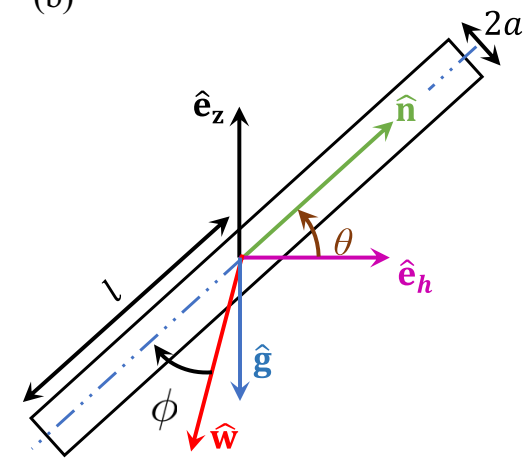

FIG. 1. Representation of the settling cylindrical particle (a) in 3D and (b) in the vertical plane ( $\hat{\mathbf{n}}, \mathbf{g})$. See text for details.

where $\mathbf{w}$ is the velocity of the center of mass. The particle Reynolds number allows us to quantify the respective role of the inertial and viscous effects in the problem. In our experiments, $\operatorname{Re}_{p}$ does not exceed 1. As a consequence, the force and the torque acting on the particle originate from the viscous term (the Stokes forces and torques, $\mathbf{f}_{S}$ and $\mathbf{T}_{S}$ ), plus contributions due to fluid inertia, which are derived in a systematic perturbation expansion in the parameter $\operatorname{Re}_{p}$, denoted here as $\mathbf{f}_{I}$ and $\mathbf{T}_{I}$.

\section{A. Translational motion}

The equations of motion for the translational degrees of freedom read as

$$
\frac{d \mathbf{x}}{d t}=\mathbf{w} \quad \text { and } \quad \frac{d \mathbf{w}}{d t}=\mathbf{g}+\frac{\mathbf{f}_{H}}{m_{p}},
$$

where $\mathbf{f}_{H} \equiv \mathbf{f}_{S}+\mathbf{f}_{I}$ is the total hydrodynamic force acting on the particle, and $m_{p}$ is its mass. The general expression for the Stokes force is: $\mathbf{f}_{S}=6 \pi a \mu \mathbb{A}(\hat{\mathbf{n}})(\mathbf{u}-\mathbf{w})$, where $\mu$ is the dynamic viscosity of the fluid, $\mathbf{u}$ is the undisturbed velocity of the fluid at the particle position (equal to zero in a quiescent flow), and $\mathbb{A}(\hat{\mathbf{n}})$ is the resistance tensor [25]. The tensor $\mathbb{A}$ can be expressed in terms of two coefficients, $A_{\perp}$ and $A_{\|}: A_{i j}=A_{\perp}\left(\delta_{i j}-n_{i} n_{j}\right)+A_{\|} n_{i} n_{j}$. The expressions of $A_{\perp}$ and $A_{\|}$depend on the shape of the particle (rod or spheroid), and in particular on its aspect ratio $\beta$, and are given in Appendix $\mathrm{A}$. In the limit $\beta \gg 1$, the expressions reduce, for the two shapes considered, to $A_{\perp}=2 A_{\|}=(4 / 3)[\beta / \log (\beta)]$. Equation (2), together with the specific form of $\mathbf{f}_{S}$, imply that if $\left|\mathbf{f}_{I}\right| \ll\left|\mathbf{f}_{S}\right|$ the particle velocity $\mathbf{w}$ relaxes with a characteristic (Stokes) time $\tau_{p}=a^{2} \log (\beta)\left(\rho_{p} / \rho_{f}\right) /(3 v)$. In our experiments, we observe that the characteristic timescales of the particles (of the order of seconds) are much longer than $\tau_{p}$ (of the order of milliseconds). This implies that the dynamics is overdamped, and that the center-of-mass velocity can be obtained by solving $\mathbf{g}+\mathbf{f}_{H} / m_{p}=0$ at any time.

The effect of finite fluid-inertia leads to a correction to the Stokes force. In the case of a spheroid, this correction to the resistance tensor can be expressed as: $\mathbb{A}^{s} \rightarrow \mathbb{A}^{s}+\mathbb{A}_{I}^{s}$. Similarly, the expressions for the corrections to the force for a slender rod, $\mathbf{f}_{I}^{r}$, can be found in Ref. [2], and are given in the Appendix A.

\section{B. Angular motion}

As stated before, in our experiments the change in the orientation of a particle is due to variations of the angle $\theta$ only. The equation of motion for this angle is obtained by projecting the equation for 
the angular momentum along the $\hat{\mathbf{p}}$ direction:

$$
I_{p} \ddot{\theta}=\left(\mathbf{T}_{S}+\mathbf{T}_{I}\right) \cdot \hat{\mathbf{p}},
$$

where $I_{p}$ is the moment of inertia of the particle with respect to its center of mass, perpendicular to its axis $\left(I_{p}=\frac{1}{12} \mathrm{ml}^{2}\right)$. As it was the case for the force, the torque can be written as the sum of a contribution due to viscous forces (Stokes), $\mathbf{T}_{S}$, and a contribution due to inertia, $\mathbf{T}_{I}$. The general expression for the Stokes torque is [26]: $\mathbf{T}_{S}=6 \pi a \mu[\mathbb{C}(\boldsymbol{\Omega}-\boldsymbol{\omega})+\mathbb{H}: \mathbb{S}]$, where $\boldsymbol{\omega}$ is the angular velocity of the solid, $\boldsymbol{\Omega}=1 / 2(\nabla \wedge \mathbf{u})$ the vorticity (which vanishes in a quiescent fluid), and $\mathbb{C}$ is the resistance tensor. The last term, $\mathbb{H}: \mathbb{S}$, involves the strain in the fluid, which vanishes in a fluid at rest, as considered here. In a quiescent fluid, the viscous torque is therefore proportional to the particle angular velocity $\omega$ which equals $\dot{\theta}$ since the $\psi$ angle is constant:

$$
T_{S} \equiv \mathbf{T}_{S} \cdot \hat{\mathbf{p}}=-C_{S} \dot{\theta},
$$

where $C_{S}$ is a resistance coefficient proportional to $\mu l^{3}$. The dimensionless constant of proportionality depends on the details of the particle. In the very large $\beta$ limit, the expression for $C_{S}$ reduces to

$$
C_{S}=\frac{8 \pi}{3} \frac{\mu l^{3}}{\log \beta} .
$$

As it was the case for the dynamics of the center of mass, the resistance term $-C_{S} \dot{\theta}$ provides a characteristic relaxation timescale for the particle orientation which is, up to a numerical factor of order 1, equal to the characteristic time $\tau_{p}$ already introduced [27]. In our experiments, the timescale over which the angle $\theta$ evolves is also found to be very long compared to $\tau_{p}$, which ensures that, to a very good approximation, the left-hand side of Eq. (3) can be set to 0 . This allows us to relate directly, via Eq. (4), the projection of the torque due to the inertial forces, $\mathbf{T}_{I}$, perpendicularly to $\hat{\mathbf{n}}$, $T_{I} \equiv \mathbf{T}_{I} \cdot \hat{\mathbf{p}}:$

$$
T_{I}=C_{S} \dot{\theta} .
$$

Determining the torque $\mathbf{T}_{I}$ is a very challenging problem, even in the small- $\operatorname{Re}_{p}$ limit.

We compare our experimental results, carried out with rods, to the results of the slender-rod theory of Ref. [2], valid asymptotically when the particle Reynolds number is small enough and for high aspect ratios $\beta$. The first criterion is very well satisfied in our experiments. The second one is not, since our particles have aspect ratios $\beta=8$ and 16, whereas the small parameter in the slender-rod theory is $1 / \log \beta$. We therefore compared also with a second perturbative theory for the torque, valid when the particle has a spheroidal shape [3]. This theory is valid for any arbitrary aspect ratio $\beta$, but only to leading order in $\operatorname{Re}_{p}$. Furthermore, it was found numerically to describe the torque acting on spheroidal particles quite well, with an accuracy of $\sim 20 \%$ for $\beta=6$ over the range of $\operatorname{Re}_{p}$ in the numerical simulations from Ref. [11]. We emphasize that the theory was derived for spheroidal particles, but we expect that it nevertheless works qualitatively for rodlike particles of the same aspect ratio and the same mass.

At small Reynolds numbers, symmetry considerations $[11,28]$ indicate that the torque takes the form

$$
\mathbf{T}_{I}=F(\beta) \rho_{f} l^{3}(\mathbf{w} \times \hat{\mathbf{n}})(\mathbf{w} \cdot \hat{\mathbf{n}}) .
$$

Equation (7) shows that $\mathbf{T}_{I}$ is along $\hat{\mathbf{p}}$, and that its norm reads

$$
T_{I}=F(\beta) \rho_{f} l^{3}|\mathbf{w}|^{2} \sin \phi \cos \phi=C_{I}|\mathbf{w}|^{2} \sin 2 \phi .
$$

The shape factor $F(\beta)$ depends only on the particle aspect ratio. As a result, the factor $C_{I}$ is known for spheroids [3] and in the slender-rod limit [2,13]; we denote its values as $C_{I}^{s}$ and $C_{I}^{r}$, respectively. The corresponding formulas are summarized in Appendix B. We note that the 


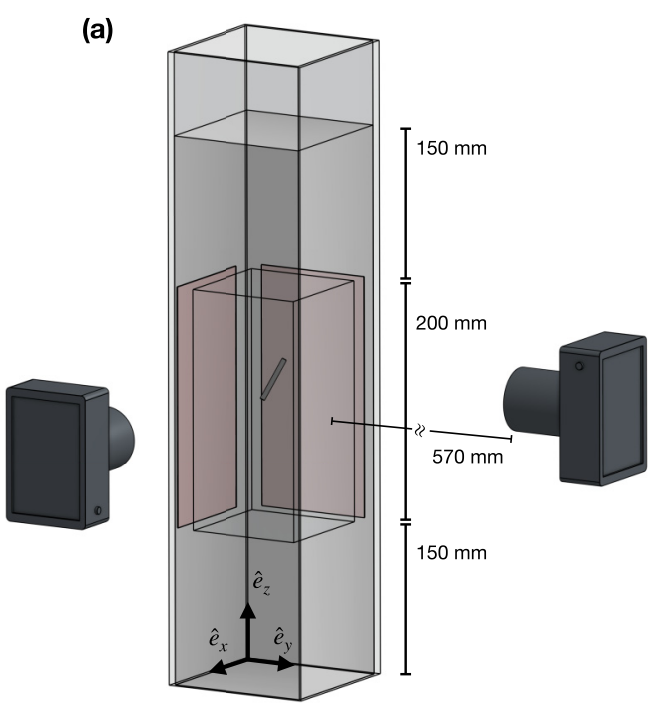

(b)

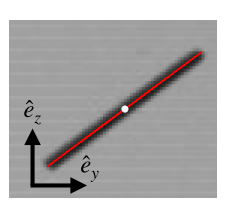

(c)

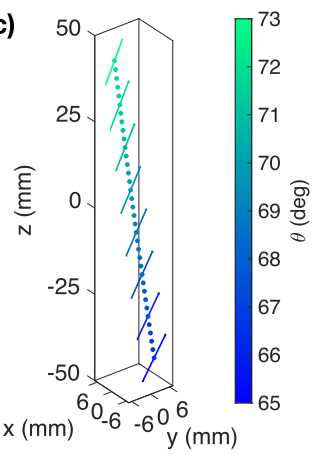

FIG. 2. (a) Schematic view of the experimental setup. The origin $(0,0,0)$ is set at the geometric center of the vessel. (b) Typical raw image obtained from one camera showing the particle of aspect ratio $\beta=16$, the detected particle center line (in red) and center of mass (white dot). (c) Successive positions of a rod's center line and center of mass during one experiment (for $\beta=16$ ), color coded by the value of the angle $\theta$. For the sake of clarity, only one center of mass data-point out of 80 , and one center line each 5 center of mass data-points are shown.

expressions for the two theories coincide in the limits $\operatorname{Re}_{p} \rightarrow 0$ and $\beta \rightarrow \infty$, where: $C_{I}(\beta) \approx$ $-5 \pi /\left[3(\log \beta)^{2}\right] \times\left(\rho_{f} l^{3} / 2\right)$. Combining Eqs. (6) and (8) leads to the prediction of the models that

$$
\dot{\theta}=\frac{C_{I}}{C_{S}}|\mathbf{w}|^{2} \sin 2 \phi
$$

This overdamped torque model for rods was found to qualitatively reproduce experimental results of rods settling in a cellular flow [13], and allowed to investigate the settling of anisotropic particles in turbulent flows [29]. The expressions of the torque for spheroids have been validated numerically [11], and used to study theoretically and numerically the settling of spheroids in turbulence $[23,24,27,30]$. We notice that when the fluid is in motion, the local velocity gradients may also contribute to the torque expression [31,32]. In our problem, with $\rho_{p} / \rho_{f} \gg 1$, the corresponding contributions are negligible.

\section{EXPERIMENTAL SETUP}

The experiments were performed in a PMMA tank with a square cross-section of side $150 \mathrm{~mm}$ and height of $710 \mathrm{~mm}$ [see Fig. 2(a)]. The tank was filled with pure glycerol (Sigma-Aldrich W252506-25KG-K), with a density $\rho_{f}=1216 \mathrm{~kg} \mathrm{~m}^{-3}$ and dynamic viscosity $\mu=1.05$ Pa.s at $22^{\circ} \mathrm{C}$ in which we observed the settling of heavy cylindrical particles. The cylindrical particles were made of Tungsten-Carbide (WC with 5\% Cobalt), manufactured by Comac Europe, with a density $\rho_{p}=14800 \mathrm{~kg} / \mathrm{m}^{3}$ resulting in a fluid-to-particle density ratio of $\rho_{p} / \rho_{f}=12.1$. Cylindrical rods were milled and filed at lengths $2 l=(16.0 \pm 0.5) \mathrm{mm}$ and $(8.0 \pm 0.5) \mathrm{mm}$, from long cylinders of diameter $2 a=(1.00 \pm 0.05) \mathrm{mm}$, resulting in cylindrical particles with aspect ratios $\beta$ of 16 and 8 . A set of 12 rods for each aspect ratio is used to avoid recovering the rods and thus perturbing the fluid at each realisation. The surface of the rods is visualized using a 
microscope, therefore ruling out fluctuations of the particles diameter larger than $0.05 \mathrm{~mm}$. As a result, the maximum dispersion in particle mass can be estimated as $\delta l / l+2 \times \delta a / a$ (where $\delta l$ and $\delta a$ are the uncertainty in particle half length and radius). This yields a maximum mass dispersion of $13 \%$ and $16 \%$, for $\beta=16$ and 8 , respectively. However, we cannot rule out the possible presence of defects in the particles, which may lead to mass inhomogeneities that would be variable from one object to the other, causing the center of mass to not coincide with the rod's geometrical center. Note that a slight variation in the center of mass position has been shown to influence the dynamics [14]. The surface roughness was measured using a Scanning Electron Microscope, leading to an average arithmetic roughness value of $15 \mu \mathrm{m}$. The particle Reynolds number $\operatorname{Re}_{p}$ was measured to lie between $0.255-0.390$ for $\beta=16$, and $0.105-0.150$ for $\beta=8$. The particles trajectories were reconstructed from images acquired using two cameras (fps1000 model from The Slow Motion Company), placed orthogonal, as shown in Fig. 2(a). The cameras acquire images at a frame rate of $1400 \mathrm{fps}$ with a resolution of $720 \times 1280 \mathrm{px}^{2}$. The volume over which the trajectories of the particles are reconstructed is $130 \times 130 \times 200 \mathrm{~mm}^{3}$ to avoid wall effects [33] - the distance between the rods and the walls is thus always larger than $10 \mathrm{~mm}$.

The particles are backlight-illuminated by using two white and homogeneous light panels [lightred rectangles in Fig. 2(a)]. A typical image captured from one camera shows the projection of the cylindrical particle in the $(y, z)$ plane in Fig. 2(b). For each image, and on both cameras, the detection of the particle is computed using the MATLAB function regionprops, which detects the particle's major axis and minor axis, center of mass and orientation [see Fig. 2(b)]. The 3D orientation of the particle is then obtained from a 3D Particle Tracking Velocimetry (PTV) algorithm [34]: a set of five points on the major axis are matched in 3D from the projections in the $(y, z)$ and $(x, z)$ planes (the five points are equally spaced between the two extrema). The particle position and orientation is thus reconstructed in 3D, as shown in Fig. 2(c), and all parameters related to the dynamics of the particles can be subsequently computed. The evolution of a typical sedimentation experiment is displayed in Fig. 2(c), from top to bottom as time increases, clearly demonstrating that the angle $\theta$ decreases in time. The tracking error of the PTV system was shown to be inferior to $130 \mu \mathrm{m}$, or equivalently $50 \%$ of the pixel size. Additionally, the raw data was filtered via the convolution with a Gaussian kernel of variance $\sigma=1.2 \times 10^{3}$ frames and $\sigma=8 \times 10^{2}$ frames, for $\beta=8$ and 16, respectively. Note that to avoid perspective distortion of the projected contour of an anisotropic object $[35,36]$, the cameras were installed sufficiently far from the sedimentation tank. An air-conditioning system keeps a constant room temperature at $(22 \pm 1)^{\circ} \mathrm{C}$, which bounds the viscosity variations to $5 \%$. Moreover, to reach thermal equilibrium, a 48-hours delay was systematically respected between the filling of the tank with pure glycerol and the experiments. The calibration of the PTV system then consisted on the displacement of a target with known dimensions over the visualization volume [34]. The particles were released in the fluid with chemical tweezers: we completely submerged the particle and released it when the glycerol's free surface was at rest (approximately after $15 \mathrm{~s}$ ). Different initial orientations covering the range $\theta(t=0) \in(0, \pi / 2)$ were imposed. A minimum time of $90 \mathrm{~s}$ was taken between two successive realisations to assure that the fluid has no motion left from the previous drop. For both $\beta=8$ and $\beta=16,25$ independent realisations have been acquired.

With the values of the physical parameters in the experiment, the characteristic timescale of the particles, $\tau_{p}$, introduced in Sec. II, does not exceed $10^{-3} \mathrm{~s}$, which, as we will document, is very short compared to the characteristic time of the evolution. Note that, additionally, there is $150 \mathrm{~mm}$ of fluid above and below the visualization volume. A particle then travels for approximately $1000 \tau_{p}$ before and after it enters the detection volume: the fluid above makes the particle loose memory of any transient produced in the release (and thereby of its initial translational and rotational velocities), whereas the fluid below keeps the particle away from the bottom-wall at all times. It cannot be excluded, however, that when the particle is injected, a weak flow, which persists as the particle settles, is induced. 

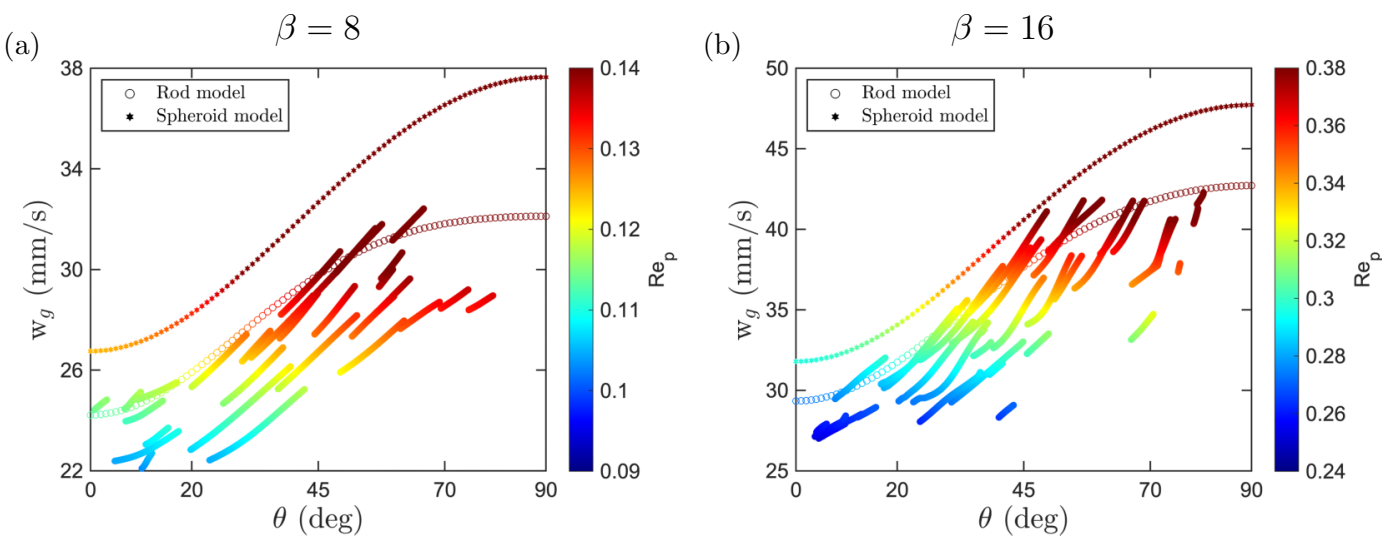

FIG. 3. Evolution of the vertical component of the velocity $\mathrm{w}_{g}$ as a function of the angle $\theta$ for (a) $\beta=8$ and (b) $\beta=16$. Each line represents one realization, color-coded by the value of $\operatorname{Re}_{p}$. Prediction for the spheroid model (stars) and for the slender-rod theory [2] (empty circles).

\section{RESULTS}

Before we proceed to present our results, we recall that the motion of the rods was found to be, to a very good approximation, planar. Namely, the variations of the angle $\psi$ between the projection of the $\mathbf{n}$ vector on a horizontal plane and an arbitrary fixed horizontal vector, as illustrated in Fig. 1(a), are less than $5^{\circ}$ during a given experiment, with a typical value of $2^{\circ}$ over all realisations. We therefore used the values of the torques valid in the case of a planar motion, where the orientation is parametrized solely by the angle $\theta$.

In the following, the scatter in the experimental data can be partially explained by the possible presence of defects in the particles, as mentioned in Sec. III, which may lead to mass inhomogeneities that would be variable from one object to the other. Recall that a set of 12 rods for each aspect ratio is used to collect our data. The data scatter is not believed to be caused by the mass dispersion between the different rods as it is also present in Ref. [14], where a smaller mass dispersion of $1 \%$ is present. The possibility of inducing a weak flow when releasing particles, already mentioned as well, represents another possible source of scatter

\section{A. Translational motion}

For all experimental realizations, the ratio between the particle inertial term $m_{p} \mathrm{~d} \mathbf{w} / \mathrm{d} t$ and the Stokes force term $\mathbf{f}_{\mathbf{S}}$ in Eq. (2) was computed to be of order $10^{-3}$. In other words, the particle inertia contribution in the translational dynamics can be neglected and the particle dynamics is overdamped, as anticipated from the ratio between $\tau_{p}$ and the characteristic time for the evolution of $\mathbf{w}$.

Figure 3 shows the settling velocity of the $\operatorname{rod~}_{g}$, defined as the projection of $\mathbf{w}$ on the vertical vector $\mathbf{g} /|\mathbf{g}|$, as a function of its orientation $\theta$, for the two values of $\beta$ considered. As for all experimental figures in this article [excluding Fig. 2(c)], each trajectory is color-coded with its instantaneous particle Reynolds number $\operatorname{Re}_{p}$ based on the particle half-length $l$. The time series of the evolution of $\theta$ are displayed in Fig. 4 and, as anticipated, we observe that the orientation of the particle, hence its velocity, evolve over a timescale which is much longer than $\tau_{p}(\sim 1 \mathrm{~ms})$. We also observe in Fig. 3 that the settling velocity is an increasing function of $\theta$ : in other words, the settling velocity increases when particles become vertical. This can be readily understood, since the drag decreases when $\theta$ increases from 0 to $90^{\circ}$. Therefore, the particle Reynolds number increases linearly with the settling velocity. The measured settling velocity is compared with the settling velocity calculated by integrating the equation of motion, Eq. (2), using for the hydrodynamic force the expressions for the spheroids model or for the slender-rod model (see Sec. II), and including the 

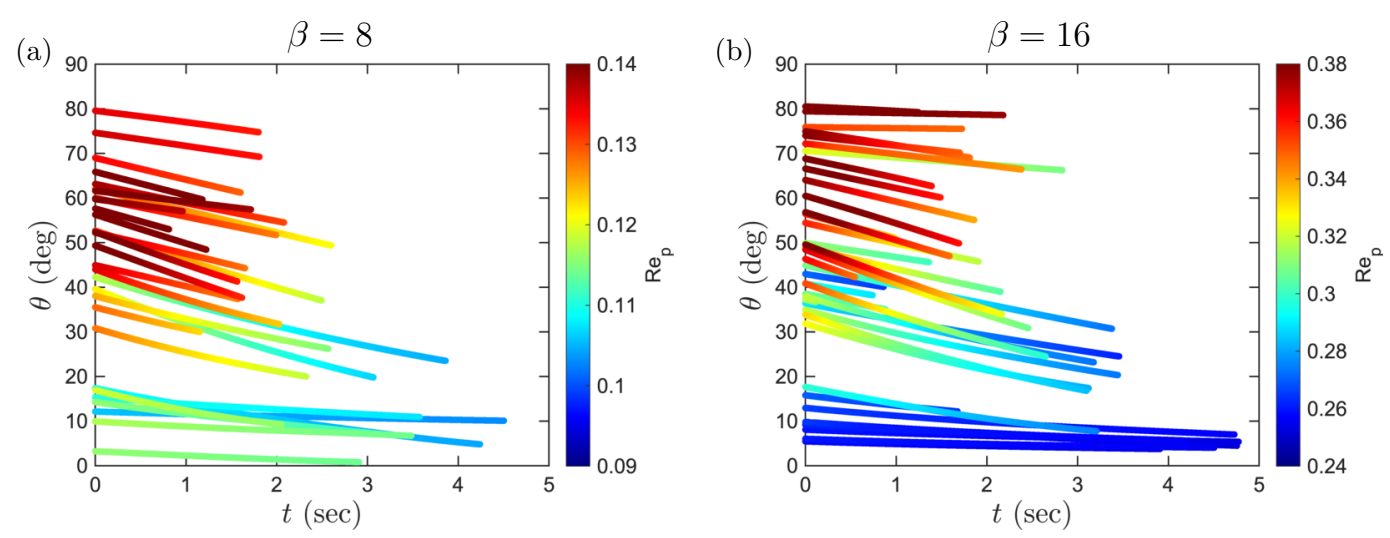

FIG. 4. Time evolution of the orientation angle $\theta$ for (a) $\beta=8$ and (b) $\beta=16$ for all realizations, colorcoded by the value of $\operatorname{Re}_{p}$.

particle inertia, despite its weak contribution in the overdamped regime. We recall here that, in the framework of the spheroid model, we use the value of the mass $m_{p}$ of the cylindrical particles used in the experiments, and not the values for a spheroid with exactly the same geometrical dimensions as that of the particles used in the experiments, for the computation of the resistance tensors $\mathbb{A}_{S}$ and $\mathbb{A}_{I}$, respectively, due to the Stokes and to the fluid inertia corrections. The velocity predicted by the spheroid model is shown as stars in Fig. 3, and the prediction from the slender-rod model is displayed as empty circles.

For the two values of $\beta$ considered, the predicted velocities overestimate the measured values, or equivalently, the predicted values of the hydrodynamic forces underestimate the actual force. However, the qualitative agreement is satisfactory: the relative difference is always smaller than $16 \%$ (for the spheroid model) and 6\% (for the slender-rod model) for $\beta=16$, and, respectively, $20 \%$ and $10 \%$ for $\beta=8$. As expected, the agreement with the predictions is better for the slender-rod model than for the spheroid model, as the particles used in the experiment have a cylindrical shape. Nonetheless, as the aspect ratio increases, the agreement improves from $\beta=8$ to $\beta=16$, even with the spheroid model. These observations can be rationalized by noticing that the sharp ends at the extrema of the cylinder play a less important role when $\beta$ increases.

The horizontal component of the measured velocity remains smaller than $20 \%$ of the vertical one, see Fig. 7 in Appendix C. The figure also shows the ratio of the horizontal and the vertical velocities predicted by the two models discussed in Sec. II for spheroids and slender-rods. It can be seen from Fig. 7 that the ratio of the experimentally measured horizontal and vertical velocities is qualitatively consistent with the predictions of the two models.

\section{B. Angular motion}

Figure 4 shows the time dependence of the angle $\theta$ for the two aspect ratios considered, for a number of different initial rod orientations. The experimental data is color-coded with the instantaneous particle Reynolds number as indicated by the color bar. The different realizations correspond to different initial orientations covering the range $\theta(t=0) \in(0, \pi / 2)$. For all realizations, $\theta$ decreases with time, meaning that particles tend to orient their broadside facing down, resulting in a maximal drag, as previously illustrated in Fig. 2(c). This trend, which has already been reported $[13,14,23,24,37,38]$, is a consequence of the action of the inertial torque $T_{I}$. Equation (8) shows, in the case of a fluid at rest, that under the effect of this torque, the particle orientation has two fixed points, a vertical one $(\theta=\pi / 2)$, and a horizontal one $(\theta=0)$, but only the latter is stable. This is consistent with the observation that in Fig. 4 the magnitude of the angular velocity reduces as the particle approaches horizontal orientation, i.e. as $\theta$ tends to 0 . 

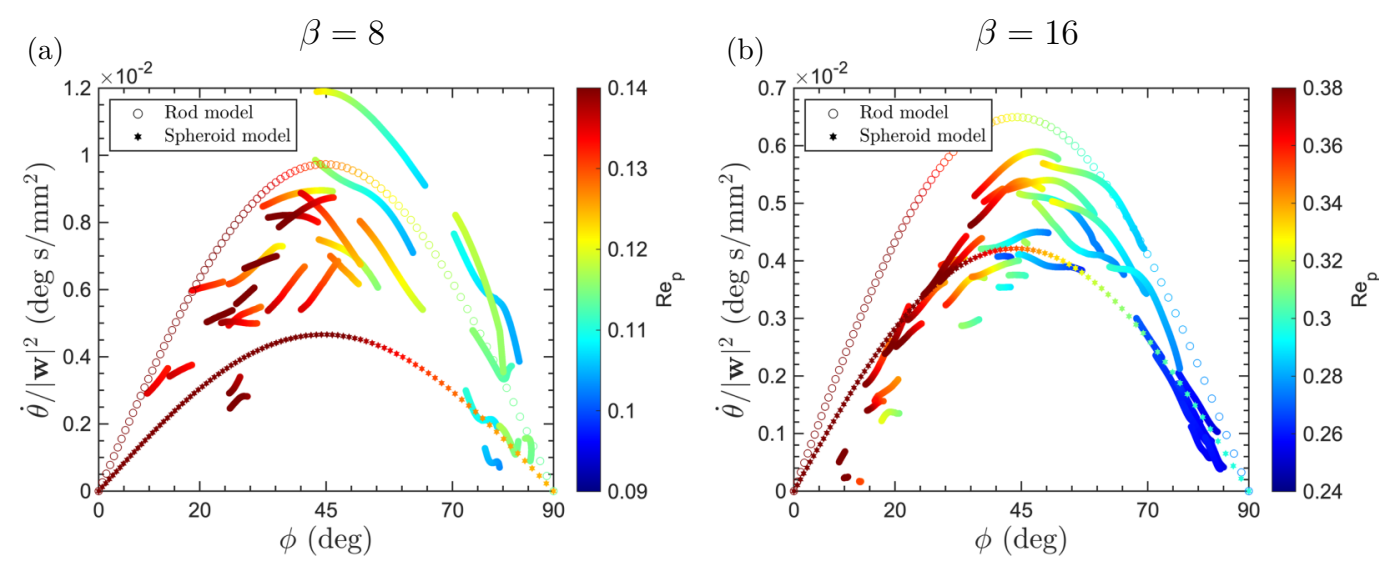

FIG. 5. The measured angular velocity, $\dot{\theta}$, divided by $|\mathbf{w}|^{2}$, color coded by the local value of $\operatorname{Re}_{p}$. The predictions of Eq. (9) are shown for the spheroid model and for the slender-rod model, as indicated by the legend. Panel (a) corresponds to $\beta=8$ and panel (b) to $\beta=16$.

Finally, it is worth mentioning that the particle Reynolds number $\operatorname{Re}_{p}$ is smaller when the particle settles horizontally $\left(\theta=0^{\circ}\right)$ as compared to vertically $\left(\theta=90^{\circ}\right)$. This is a consequence of the fact that the drag exerted on a fiber settling broadside down is higher than that on a fiber settling with its narrow edge first. The later results in a velocity increase with $\theta$, as seen in Fig. 3 .

We now estimate the importance of the particle inertia term in the rotational dynamics, Eq. (3), from the ratio $I_{p} \ddot{\theta} / C_{S} \dot{\theta}$. This ratio is very small, well below $10^{-3}$, for the two $\beta$ values. As a consequence, the left-hand side term of Eq. (3) is negligible with respect to either of the two terms on the right-hand side. In other words, the particle inertia contribution in the rotational dynamics can be neglected: the fluid-inertia torque $\left(T_{I}\right)$ and the Stokes torque $\left(T_{S}\right)$ essentially balance each other, so the particle angular dynamics is overdamped. Neglecting the second order derivative in Eq. (3) allows us to simply relate the torque $T_{I}$ to the instantaneous rotation rate, $\dot{\theta}$, which therefore provides us with an elementary way to determine $T_{I}$. To compare the results of the experiments with the prediction of Eq. (9), Fig. 5 shows, for $\beta=8$ (panel a) and for $\beta=16$ (panel b), the measured ratio $\dot{\theta} /|\mathbf{w}|^{2}$ as a function of the pitch angle, $\phi$. The trajectories are color coded by the recorded value of the particle Reynolds number, $\mathrm{Re}_{p}$, as indicated by the color bars. Equation (9) predicts that the points should be along a curve $\dot{\theta} /|\mathbf{w}|^{2}=\left(C_{I} / C_{S}\right) \sin 2 \phi$. The expected values for rods (empty circles) and for spheroids (stars) are indicated in the figure. We also tested the torque expression proposed by Ref. [12]: it leads in the regime of our experiments $\left(\operatorname{Re}_{\mathrm{p}}<0.4\right)$ to curves virtually indistinguishable from those plotted by using the slender-rod model from Ref. [2] (empty circles). As shown in Fig. 5, the constants $C_{I}$ and $C_{S}$ for rods and spheroids differ by a factor $\approx 2$ for $\beta=8$, and $\approx 1.5$ for $\beta=16$.

The dependence of $\dot{\theta} /|\mathbf{w}|^{2}$ qualitatively follows the $\sin 2 \phi$ prediction, although with a very large dispersion, particularly for $\beta=8$. Interestingly, for both $\beta=8$ and $\beta=16$, the slender-rod theory overpredicts the value of $\dot{\theta} /|\mathbf{w}|^{2}$. However, the spheroid theory underpredicts the observed dependence, although the agreement becomes better when $\beta=16$.

In view of the limitations of the theory, it is reassuring to see that the theoretical predictions provide the right order of magnitude for the experimental results. To discuss further, we recall that the two theories used here for comparison have different shortcomings. Namely, the slender-rod theory (open circles) is valid over a larger range of Reynolds numbers, but the theory rests on an expansion in $1 / \log \beta$, and is therefore valid only when $\log \beta \gg 1$, a condition which is in practice extremely difficult to satisfy. However, the theory for spheroids works for any aspect ratio ( $\beta$ does not need to be very large), but the predictions of the torque were shown to deviate by $\sim 30 \%$ for values of $\operatorname{Re}_{p}=0.3$ [11], and deviations grow when $\operatorname{Re}_{p}$ in- 
TABLE I. Comparison of the parameters of the present work and of Ref. [14].

\begin{tabular}{lllllr}
\hline \hline & & $\mathrm{Re}_{\mathrm{p}}$ & $\mathrm{Re}_{\mathrm{D}}$ & $\mathrm{Re}_{\mathrm{D}} / \beta^{2}$ & $\rho_{p} / \rho_{f}$ \\
\hline Present work & $\beta=8$ & 0.15 & 0.037 & $6 \times 10^{-4}$ & 12 \\
& $\beta=16$ & 0.4 & 0.05 & $2 \times 10^{-4}$ & \\
Roy et al. [14] & $\beta=20$ & 1.6 & 0.16 & $4 \times 10^{-4}$ & 3 \\
& $\beta=100$ & 8.6 & 0.17 & $1.7 \times 10^{-5}$ & \\
\hline \hline
\end{tabular}

creases. In this context, our observations fall in the delicate regime where both theories have shortcomings.

The prediction for spheroids largely underestimates our measurements for $\beta=8$, but the agreement is much better for $\beta=16$, as expected since the exact shape of the particle extrema obviously plays a less important role at larger aspect ratio. The slender-rod theory overestimates the experimental values by roughly $20 \%$ for the two values of $\beta$ considered. Such a result can be interpreted by comparing it with those of previous experimental work [14].

For the sake of such a comparison, it is particularly useful to recall the observation of Ref. [12], suggesting that the approximation of the torque using slender body theory [2] is accurate for cylindrical particle kept fixed in a uniform flow, under the conditions that $\operatorname{Re}_{D} \ll 1$ and that the ratio $\operatorname{Re}_{D} / \beta^{2}$ is smaller than a very small value, where $\operatorname{Re}_{D}$ is the particle Reynolds number based on its diameter $\left[\operatorname{Re}_{D}=\operatorname{Re}_{p} \times(2 / \beta)\right.$ with our notations]. In their numerical setup, the authors of Ref. [12] find that the ratio $\operatorname{Re}_{D} / \beta^{2}$ has to be smaller than 0.005 for the theory to describe the data. The theoretical prediction fails to reproduce the data when $\operatorname{Re}_{D} / \beta^{2}$ is larger than $\sim 0.01$. The values of the two particle Reynolds numbers and of this parameter obtained in the present investigation and in Ref. [14] are reported in Table I. The slender-rod theory correctly predicts the torque in Ref. [14] for $\beta=100$, but significantly overestimates it in the three other configurations. Table I shows that, as already discussed, our Reynolds numbers $\operatorname{Re}_{p}$ and $\operatorname{Re}_{D}$ are significantly smaller than those of Roy et al. However, $\operatorname{Re}_{D}$ is smaller than 0.18 in the four experiments. Interestingly, the parameter $\operatorname{Re}_{D} / \beta^{2}$ is of order $2 \times 10^{-5}$ in the only configuration correctly predicted by the theory $(\beta=100$ in Ref. [14]), and larger than $10^{-4}$ in the three others. These results are therefore compatible with the fact that the conditions under which the asymptotic theory [2] holds are: $\operatorname{Re}_{D} \ll 1$ and $\operatorname{Re}_{D} / \beta^{2} \ll 1$. Thus, for the present experiment, the threshold for the second criterion seems to be $\sim 10^{-4}$, a value smaller than that obtained numerically by Ref. [12]. A likely reason for this difference is that in the numerical setup the particle is kept fixed with respect to the flow, whereas its orientation is time-dependent in the experiments.

\section{CONCLUSIONS}

In this work, we studied experimentally the dynamics of rods settling in a quiescent flow. Our experiments were in the regime where the angular dynamics is overdamped, so the torque and the force acting on the object could be readily determined from the settling and from the angular velocities, which was made possible via a 3D-PTV. We considered two particles, with aspect ratios $\beta=8$ and $\beta=16$. In the two cases, the Reynolds numbers were small, making comparisons with predictions at low particle Reynolds numbers $\left(\operatorname{Re}_{\mathrm{p}} \ll 1\right)$ meaningful. Our measurements present a nonnegligible scatter that is hypothesised to be caused by particle mass inhomogeneity and/or spurious flows.

We compared the measured torques with theoretical predictions in the slender-rod limit, and in the case of spheroids. Our results show that both models qualitatively predict the translational dynamics, in particular the slender-rod theory was found to represent better the experimental data.

Regarding the rod angular dynamics, the models simplified as the rotational dynamics was found to be planar and overdamped. The particles were seen to orient broadside on, i.e. with the maximal drag orientation. 
The torque is found to be qualitatively well described by a $\sin 2 \phi$ functional form, and differences in the torque prefactor that both models provide were seen. The spheroid theory was found to describe correctly the dynamics of the rods with $\beta=16$, whereas for the case $\beta=8$ the agreement is less satisfactory, the experimental prefactor being roughly twice as large as the theory predictions. The slender rod theory overestimates the experimental data by $\sim 20 \%$ for the two aspect ratios considered. Combining the data from Ref. [14] and ours confirms the intuition from Ref. [12] according to which the slender-rod theory [2] is valid if $\operatorname{Re}_{D} \ll 1$ and $\operatorname{Re}_{D} / \beta^{2}$ smaller than a very small quantity, where $\operatorname{Re}_{D}$ is the particle Reynolds number based on its diameter: the ratio $\operatorname{Re}_{D} / \beta^{2}$ is of order $2 \times 10^{-5}$ in the unique configuration correctly predicted by the theory, while it is $>10^{-4}$ in all other cases. This threshold value is smaller than the one obtained by Ref. [12] $(\sim 0.005-0.01)$ : this difference may be attributed to the fact that in this numerical investigation the orientation of the particles is fixed with respect to the flow, whereas its orientation is time-dependent in the experiments.

All in all, a value of $\operatorname{Re}_{D} \sim 0.15$ seems to be small enough for the slender-rod theory to hold, provided that the particle aspect ratio is high enough, a regime difficult to reach in practical situations. Providing an exact expression of the torque acting on an anisotropic body is a very difficult issue.

\section{ACKNOWLEDGMENTS}

The authors are grateful to Jacques Magnaudet for enlightening discussions and to Vincent Dolique for his help with the Scanning Electron Microscopy. This work was supported by the French research program IDEX-LYON of the University of Lyon in the framework of the French program "Programme Investissements d'Avenir" (Grant No. hlR-16-IDEX-0005). This research was also supported by Higher Education Commission Pakistan and Campus France.

\section{APPENDIX A: EXPRESSION FOR THE FORCE ACTING ON ANISOTROPIC PARTICLES}

We begin by recalling the general expression for the Stokes force [39]:

$$
\mathbf{f}_{S}=6 \pi \mu a \mathbb{A}(\hat{\mathbf{n}})(\mathbf{u}-\mathbf{w}),
$$

where the components of the tensor $\mathbb{A}$ are given by $A_{i j}=A_{\|} n_{i} n_{j}+A_{\perp}\left(\delta_{i j}-n_{i} n_{j}\right)$. The expressions of $A_{\|}$and $A_{\perp}$ are, in the case of a spheroid:

$$
A_{\|}^{s}=\frac{8}{3} \frac{\beta}{\chi_{0}+\beta^{2} \gamma_{0}} \text { and } A_{\perp}^{s}=\frac{8}{3} \frac{\beta}{\chi_{0}+\alpha_{0}},
$$

with

$$
\alpha_{o}=\frac{\beta^{2}}{\beta^{2}-1}-\beta \frac{\cosh ^{-1} \beta}{\left(\beta^{2}-1\right)^{3 / 2}}, \quad \gamma_{o}=\frac{-2}{\beta^{2}-1}+2 \beta \frac{\cosh ^{-1} \beta}{\left(\beta^{2}-1\right)^{3 / 2}}, \quad \text { and } \quad \chi_{0}=2 \beta \frac{\cosh ^{-1} \beta}{\left(\beta^{2}-1\right)^{1 / 2}} \text {. }
$$

In the case of a slender rod, the expression of the coefficients $A_{\perp}^{r}$ and $A_{\|}^{r}$ are [40]

$$
A_{\|}^{r}=\frac{2}{3} \frac{\beta}{\log \beta} \text { and } A_{\perp}^{r}=\frac{4}{3} \frac{\beta}{\log \beta} .
$$

The correction to the force due to inertial effects has been derived for spheroids in Ref. [41]. The expression for the coefficients $A_{I, \perp}^{s}$ and $A_{I, \|}^{s}$ are

$$
A_{I, \|}^{s}=\left[3 A_{\|}-\left(A_{\|} \cos ^{2} \phi+A_{\perp} \sin ^{2} \phi\right)\right] A_{\|} \text {and } A_{I, \perp}^{s}=\left[3 A_{\perp}-\left(A_{\|} \cos ^{2} \phi+A_{\perp} \sin ^{2} \phi\right)\right] A_{\perp},
$$

where $\phi=\cos ^{-1}(\hat{\mathbf{w}} \cdot \hat{\mathbf{n}})$. 


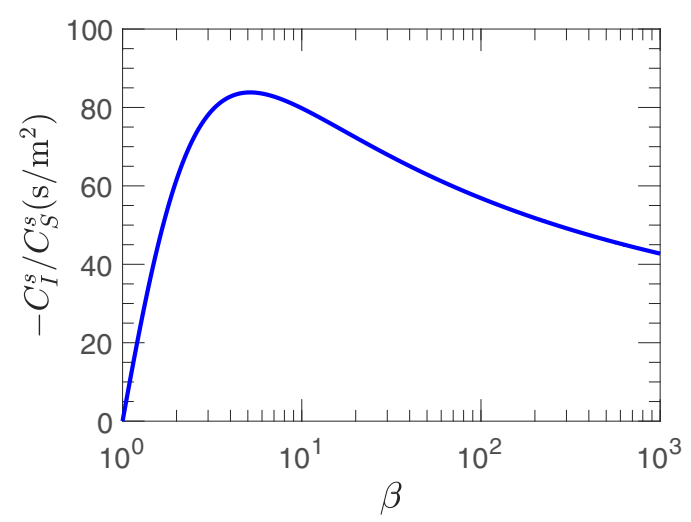

FIG. 6. The ratio $-C_{I}^{s} / C_{S}^{s}$ in the case of spheroids, plotted as a function of $\beta$ at $v=1.05 / 1216 \mathrm{~m}^{2} \mathrm{~s}^{-1}$ (the kinematic viscosity of glycerol at $22^{\circ} \mathrm{C}$ ).

In the case of a slender rod, the fluid-inertia correction to the force, $\mathbf{f}_{I}^{r}$, is given by [2]

$$
\begin{aligned}
\frac{\mathbf{f}_{I}^{r}}{2 \pi \mu|\mathbf{w}| l}= & \left(\frac{1}{\log \beta}\right)^{2}\left(\frac { 2 \operatorname { c o s } \phi \hat { \mathbf { w } } - ( 2 - \operatorname { c o s } \phi + \operatorname { c o s } ^ { 2 } \phi ) \mathbf { n } } { 2 \operatorname { R e } _ { p } ( 1 - \operatorname { c o s } \phi ) } \left\{E_{1}\left[\operatorname{Re}_{p}(1-\cos \phi)\right]\right.\right. \\
& \left.+\log \left[\operatorname{Re}_{p}(1-\cos \phi)\right]+\gamma-\operatorname{Re}_{p}(1-\cos \phi)\right\}-\frac{2 \cos \phi \hat{\mathbf{w}}-\left(2+\cos \phi+\cos ^{2} \phi\right) \mathbf{n}}{2 \operatorname{Re}_{p}(1+\cos \phi)} \\
& \times\left\{E_{1}\left[\operatorname{Re}_{p}(1+\cos \phi)\right]+\log \left[\operatorname{Re}_{p}(1+\cos \phi)\right]+\gamma-\operatorname{Re}_{p}(1+\cos \phi)\right\} \\
& -[\cos \phi \mathbf{n}-2 \hat{\mathbf{w}}]\left\{E_{1}\left[\operatorname{Re}_{p}(1-\cos \phi)\right]+\log (1-\cos \phi)+E_{1}\left[\operatorname{Re}_{p}(1+\cos \phi)\right]\right. \\
& \left.+\log (1+\cos \phi)+\frac{1-e^{-\operatorname{Re}_{p}(1-\cos \phi)}}{\operatorname{Re}_{p}(1-\cos \phi)}+\frac{1-e^{-\operatorname{Re}_{p}(1+\cos \phi)}}{\operatorname{Re}_{p}(1+\cos \phi)}+2\left[\gamma+\log \left(\operatorname{Re}_{p} / 4\right)\right]\right\} \\
& +3 \cos \phi \mathbf{n}-2 \hat{\mathbf{w}}),
\end{aligned}
$$

where $\hat{\mathbf{w}}$ is the unit vector along fiber velocity $\mathbf{w}, E_{1}(X)=\int_{X}^{+\infty} \frac{e^{-t}}{t} d t$, and $\gamma$ is the Euler constant.

\section{APPENDIX B: EXPRESSION FOR THE TORQUE ACTING ON ANISOTROPIC PARTICLES}

The crucial parameter for our work is the ratio $C_{I} / C_{S}$ in Eq. (9), where $C_{S}$ and $C_{I}$ are defined by Eqs. (4) and (8), respectively.

\section{Spheroids}

In the case of spheroids, the shape factor, $F(\beta)$, has been determined in Ref. [3] [see, in particular, Eq. (4.1)]. The function $F(\beta)$ is represented, e.g., in Fig. 1 of Ref. [11]. In the limit of very large aspect ratio, $\beta \gg 1, F(\beta) \approx-5 \pi /\left[3(\log \beta)^{2}\right]$.

The ratio between the two coefficients $C_{I}^{s}$ and $C_{S}^{s}$, appearing in Eq. (9), is independent of particle size and only depends on $\beta$ and $\nu$; it is given as

$$
\frac{C_{I}^{s}}{C_{S}^{s}}=\frac{\rho_{f} l^{3} F(\beta)}{2} \frac{3}{16} \frac{1}{\pi \mu a^{3} \beta}\left(\frac{\alpha_{o}+\beta^{2} \gamma_{o}}{1+\beta^{2}}\right)=\frac{3}{32} \frac{\beta^{2} F(\beta)}{\pi v}\left(\frac{\alpha_{o}+\beta^{2} \gamma_{o}}{1+\beta^{2}}\right)
$$

The ratio in Eq. (B1) is plotted in Fig. 6. 


\section{Slender rods}

In the case of rods, the expression for the torque $T_{I}^{r}$ is given by [2]

$$
T_{I}^{r}=H\left(\operatorname{Re}_{p}, \phi\right)|\mathbf{w}| \sin 2 \phi=C_{I}^{r}|\mathbf{w}|^{2} \sin 2 \phi,
$$

where $C_{I}^{r}=H\left(\operatorname{Re}_{\mathrm{p}}, \phi\right) /|\mathbf{w}|$ with the following expression for $H$ :

$$
\begin{aligned}
\frac{(\log \beta)^{2}}{2 \pi \mu l^{2}} H\left(\operatorname{Re}_{\mathrm{p}}, \phi\right)= & \frac{1}{2 \operatorname{Re}_{p}(1-\cos \phi)}\left\{2+2 \frac{e^{-\operatorname{Re}_{p}(1-\cos \phi)}-1}{\operatorname{Re}_{p}(1-\cos \phi)}-E_{1}\left[\operatorname{Re}_{p}(1-\cos \phi)\right]\right. \\
& \left.-\log \left[\operatorname{Re}_{p}(1-\cos \phi)\right]-\gamma\right\}+\frac{1}{2 \operatorname{Re}_{p}(1+\cos \phi)}\left\{2+2 \frac{e^{-\operatorname{Re}_{p}(1+\cos \phi)}-1}{\operatorname{Re}_{p}(1+\cos \phi)}\right. \\
& \left.-E_{1}\left[\operatorname{Re}_{p}(1+\cos \phi)\right]-\log \left[\operatorname{Re}_{p}(1+\cos \phi)\right]-\gamma\right\}-\frac{1}{\operatorname{Re}_{p}(1-\cos \phi) \cos \phi} \\
& \times\left\{1-\frac{1-e^{-\operatorname{Re}_{p}(1-\cos \phi)}}{\operatorname{Re}_{p}(1-\cos \phi)}\right\}+\frac{1}{\operatorname{Re}_{p}(1+\cos \phi) \cos \phi} \\
& \times\left\{1-\frac{1-e^{-\operatorname{Re}_{p}(1+\cos \phi)}}{\operatorname{Re}_{p}(1+\cos \phi)}\right\} .
\end{aligned}
$$

In the limit $\operatorname{Re}_{p} \rightarrow 0$, appropriate in the case of our study, and for $\beta \gg 1$, we find that

$$
T_{I}^{r} \approx-\frac{5 \pi}{6(\log \beta)^{2}} \rho_{f}|\mathbf{w}|^{2} l^{3} \sin 2 \phi,
$$

which coincides with the expression derived in Ref. [3]. The ratio $C_{I}^{r} / C_{S}^{r}$ for slender rods reads

$$
\frac{C_{I}^{r}}{C_{S}^{r}}=\frac{H\left(\operatorname{Re}_{p}, \phi\right)}{|\mathbf{w}|} \frac{3}{8 \pi} \frac{\log \beta}{\mu l^{3}}=\frac{3}{8 \pi} \frac{H\left(\operatorname{Re}_{p}, \phi\right) \log \beta}{|\mathbf{w}| \mu l^{3}} .
$$

For a particle settling in a viscous fluid, the ratio in Eq. (B5) is a function of particle orientation through the particle Reynolds number $\mathrm{Re}_{p}$, the settling velocity $\mathbf{w}$, and the pitch angle $\phi$. The averaged values (over all orientations) of $C_{I}^{r} / C_{S}^{r}$ for the cylindrical particles under consideration are -170 and -115 for $\beta=8$ and $\beta=16$, respectively. The corresponding values in case of spheroids are -82 and -75 , see Fig. 6 .
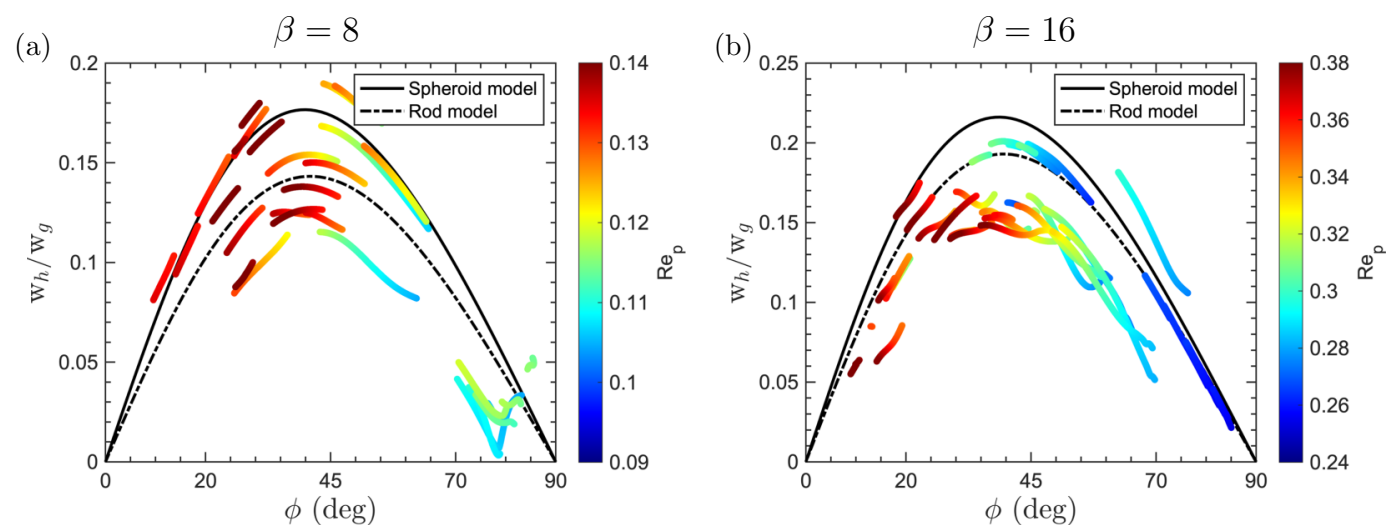

FIG. 7. Ratio between horizontal $\left(\mathrm{w}_{h}\right)$ and vertical $\left(\mathrm{w}_{g}\right)$ velocity components, as a function of $\phi$. 


\section{APPENDIX C: HORIZONTAL TRANSLATIONAL DYNAMICS}

Figure 7 shows the ratio between the horizontal, $\mathrm{w}_{h}$, and the vertical, $\mathrm{w}_{g}$, components of velocity. We also show the expected ratios from the spheroid model (full line) and from the slender-rod model (dashed-dotted lines), for $\beta=8$ [Fig. 7(a)] and for $\beta=16$ [Fig. 7(b)]. The ratios are all found to be small, less than $\sim 20 \%$, in agreement with the predictions from the two models, which differ by no more than $\sim 20 \%$ from each other.

[1] R. G. Cox, The steady motion of a particle of arbitrary shape at small Reynolds numbers, J. Fluid Mech. 23, 625 (1965).

[2] R. E. Khayat and R. G. Cox, Inertia effects on the motion of long slender bodies, J. Fluid Mech. 209, 435 (1989).

[3] V. Dabade, N. K Marath, and G. Subramanian, Effects of inertia and viscoelasticity on sedimenting anisotropic particles, J. Fluid Mech. 778, 133 (2015).

[4] A. Hölzer and M. Sommerfeld, Lattice Boltzmann simulations to determine drag, lift, and torque acting on nonspherical particles, Comput. Fluids 38, 572 (2009).

[5] M. Zastawny, G. Mallouppas, F. Zhao, and B. van Wachem, Derivation of drag and lift forces and torque coefficients for nonspherical particles in a flow, Int. J. Multiphase Flow 39, 227 (2012).

[6] F. Jiang, J. P. Gallardo, and H. I. Andersson, The laminar wake behind a 6:1 prolage spheroid at $45^{\circ}$ incidence angle, Phys. Fluids 26, 113602 (2014).

[7] R. Ouchene, M. Khalij, A. Taniére, and B. Arcen, Lattice Boltzmann simulations to determine drag, lift, and torque acting on nonspherical particles, Comput. Fluids 113, 53 (2015).

[8] R. Ouchene, M. Khalij, B. Arcen, and A. Taniére, A new set of correlations of drag, lift and torque coefficients for nonspherical particles at large Reynolds numbers, Powder Technol. 303, 33 (2016).

[9] H. I. Andersson and F. Jiang, Forces and torques on a prolate spheroid: Low-Reynolds number and attack angle effects, Acta Mech. 230, 431 (2019).

[10] K. Fröhlich, M. Meinke, and W. Schröder, Derivation of drag and lift forces and torque coefficients for nonspherical particles in a flow, J. Fluid Mech. 901, A5 (2020).

[11] F. Jiang, L. Zhao, H. Andersson, K. Gustavsson, A. Pumir, and B. Mehlig, Inertial torque on a small spheroid in a uniform flow, Phys. Rev. Fluids 6, 024302 (2021).

[12] M. Kharrouba, J.-L. Pierson, and J. Magnaudet, Flow structure and loads over inclined cylindrical rodlike particles and fibers, Phys. Rev. Fluids 6, 044308 (2021).

[13] D. Lopez and E. Guazzelli, Inertial effects on fibers settling in a vortical flow, Phys. Rev. Fluids 2, 024306 (2017).

[14] A. Roy, R. J. Hamati, L. Tierney, D. L. Koch, and G. A. Voth, Inertial torques and a symmetry breaking orientational transition in the sedimentation of slender fibres, J. Fluid Mech. 875, 576 (2019).

[15] F. Lundell, L. D. Söderberg, and P. H. Alfredsson, Fluid mechanics of papermaking, Ann. Rev. Fluid Mech. 43, 195 (2011).

[16] W. M. Durham, E. Climent, M. Barry, F. De Lillo, G. Boffetta, M. Cencini, and R. Stocker, Turbulence drives microscale patches of motile phytoplankton, Nat. Commun. 4, 2148 (2013).

[17] T. J. Pedley and J. O. Kessler, Hydrodynamic phenomena in suspensions of swimming microorganisms, Ann. Rev. Fluid Mech. 24, 313 (1992).

[18] J. Ruiz, D. Macías, and F. Peters, Turbulence increases the average settling velocity of phytoplankton cells, Proc. Natl. Acad. Sci. USA 101, 17720 (2004).

[19] H. R. Pruppacher and J. D. Klett, Microphysics of clouds and precipitation, Nature (London) 284, 88 (1980).

[20] C. Siewert, R. P. J. Kunnen, M. Meinke, and W. Schröder, Orientation statistics and settling velocity of ellipsoids in decaying turbulence, Atmos. Res. 142, 45 (2014).

[21] K. Gustavsson, J. Jucha, A. Naso, E. Lévêque, A. Pumir, and B. Mehlig, Statistical Model for the Orientation of Nonspherical Particles Settling in Turbulence, Phys. Rev. Lett. 119, 254501 (2017). 
[22] J. Jucha, A. Naso, E. Lévêque, and A. Pumir, Settling and collision between small ice crystals in turbulent flows, Phys. Rev. Fluids 3, 014604 (2018).

[23] K. Gustavsson, M. Z. Sheikh, D. Lopez, A. Naso, A. Pumir, and B. Mehlig, Effect of fluid inertia on the orientation of a small prolate spheroid settling in turbulence, New J. Phys. 21, 083008 (2019).

[24] M. Z. Sheikh, K. Gustavsson, D. Lopez, E. Lévèque, B. Mehlig, A. Pumir, and A. Naso, Importance of fluid inertia for the orientation of spheroids settling in turbulent flow, J. Fluid Mech. 886, A9 (2020).

[25] Sangtae Kim and Seppo J. Karrila, Microhydrodynamics: Principles and Selected Applications (Butterworth-Heinemann, Boston, MA, 1991).

[26] G. B. Jeffery, The motion of ellipsoidal particles immersed in a viscous fluid, Proc. R. Soc. London. Ser. A 102, 161 (1922).

[27] P. Anand, S. S. Ray, and G. Subramanian, Orientation Dynamics of Sedimenting Anisotropic Particles in Turbulence, Phys. Rev. Lett. 125, 034501 (2020).

[28] G. Subramanian and D. L. Koch, Inertial effects on fibre motion in simple shear flow, J. Fluid Mech. 535, 383 (2005).

[29] S. Kramel, Non-Spherical Particle Dynamics in Turbulence, Ph.D. thesis, Wesleyan College, 2017.

[30] K. Gustavsson, M. Z. Sheikh, A. Naso, A. Pumir, and B. Mehlig, Effect of particle inertia on the alignment of small ice crystals in turbulent clouds, J. Atmos. Sci. 78, 2573 (2021).

[31] J. Einarsson, F. Candelier, F. Lundell, J. R. Angilella, and B. Mehlig, Rotation of a spheroid in a simple shear at small Reynolds number, Phys. Fluids 27, 063301 (2015).

[32] F. Candelier, B. Mehlig, and J. Magnaudet, Time-dependent lift and drag on a rigid body in a viscous steady linear flow, J. Fluid Mech. 864, 554 (2019).

[33] R. P. Chhabra, S. Agarwal, and K. Chaudhary, A note on wall effect on the terminal falling velocity of a sphere in quiescent Newtonian media in cylindrical tubes, Powder Technol. 129, 53 (2003).

[34] M. Bourgoin and S. G. Huisman, Using ray-traversal for 3D particle matching in the context of particle tracking velocimetry in fluid mechanics, Rev. Sci. Instr. 91, 085105 (2020).

[35] J. B. Huang, Z. Chen, and T. L. Chia, Pose determination of a cylinder using reprojection transformation, Pattern Recogn. Lett. 17, 1089 (1996).

[36] C. Toupoint, P. Ern, and V. Roig, Kinematics and wake of freely falling cylinders at moderate Reynolds numbers, J. Fluid Mech. 866, 82 (2019).

[37] K. O. L. F. Jayaweera and B. J. Mason, The behaviour of freely falling cylinders and cones in a viscous fluid, J. Fluid Mech. 22, 709 (1965).

[38] G.M. Bragg, L. van Zuiden, and C.E. Hermance, The free fall of cylinders at intermediate Reynold's numbers, Atmos. Environ. (1967) 8, 755 (1974).

[39] A. Oberbeck, Über stationäre Flüssigkeitsbewegungen mit Berücksichtigung der inneren Reibung, J. Reine Angew. Math. 81, 62 (1876).

[40] G. K. Batchelor, Slender-body theory for particles of arbitrary cross-section in stokes flow, J. Fluid Mech. 44, 419 (1970).

[41] H. Brenner, The Oseen resistance of a particle of arbitrary shape, J. Fluid Mech. 11, 604 (1961). 